\title{
RESOLVENT ESTIMATES AND RESONANCE FREE DOMAINS FOR SCHRÖDINGER OPERATORS WITH MATRIX-VALUED POTENTIALS
}

\author{
MAROUANE ASSAL
}

\begin{abstract}
We establish semiclassical resolvent estimates for Schrödinger operators with long-range matrix-valued potentials. As an application we prove resonance free domains both in trapping and non-trapping situations. Our results generalize the well-known results of $[\mathrm{Bu}, \mathrm{Ma}]$ in the case of scalar Schrödinger operators.
\end{abstract}

\section{INTRODUCTION AND BACKGROUND}

We are interested in resolvent estimates and quantum resonances for Schrödinger operators with long-range matrix-valued potentials in the semiclassical regime. The results established here are a part of a work in progress [As] where we will give some applications to scattering theory for matrix Schrödinger operators.

1.1. Preliminaries. Consider the semiclassical Schrödinger operator on the Hilbert space $L^{2}\left(\mathbb{R}^{d} ; \mathbb{C}^{N}\right), d \geq 1$

$$
P(h):=-h^{2} \Delta \cdot I_{N}+V(x),
$$

where $I_{N}$ is the identity $N \times N$ matrix and $V: \mathbb{R}^{d} \rightarrow \mathcal{H}_{N}$ is a smooth $N \times N$ hermitian matrix-valued potential, i.e.,

$$
V(x)=\left(V_{i j}(x)\right)_{1 \leq i, j \leq N}, \quad V_{i j}(x)=\overline{V_{j i}(x)},
$$

with long-range behavior at infinity, i.e., there exists a constant matrix $V_{\infty} \in \mathcal{H}_{N}$ and $\rho_{0}>0$ such that

$$
\left\|\partial_{x}^{\alpha}\left(V(x)-V_{\infty}\right)\right\|_{N \times N}=\mathcal{O}_{\alpha}\left(\langle x\rangle^{-\rho_{0}-|\alpha|}\right), \quad \forall x \in \mathbb{R}^{d}, \alpha \in \mathbb{N}^{d},
$$

with $\langle x\rangle:=\left(1+|x|^{2}\right)^{1 / 2}$. Here $\mathcal{H}_{N}$ denotes the space of $N \times N$ hermitian matrices endowed with the norm $\|\cdot\|_{N \times N}$ defined by (2.1) and $h>0$ is the semiclassical parameter. Such operators arise as important models in molecular physics and quantum chemistry, for instance in the Born-Oppenheimer approximation which allows for a drastic reduction of problem size when dealing with molecular systems. In this case, the semiclassical parameter $h$ represents the square root of the quotient between the electronic and nuclear masses (see e.g. [KMSW, Je1]).

Set $P_{\infty}(h):=-h^{2} \Delta \cdot I_{N}+V_{\infty}$ and we denote $\lambda_{1} \leq \cdots \leq \lambda_{N}$ the eigenvalues of the constant matrix $V_{\infty}$. Without loss of generality, we can assume that $V_{\infty}$ is diagonal, i.e.,

$$
V_{\infty}=\operatorname{diag}\left(\lambda_{1}, \ldots, \lambda_{N}\right) .
$$

Key words and phrases. Matrix Schrödinger operators, semiclassical resolvent estimates, Carleman estimates, resonance free domains. 
The operator $P_{\infty}(h)$ is self-adjoint in $L^{2}\left(\mathbb{R}^{d} ; \mathbb{C}^{N}\right)$ with domain the Sobolev space $H^{2}\left(\mathbb{R}^{d} ; \mathbb{C}^{N}\right)$, and its spectrum coincides with $\left[\lambda_{1},+\infty\right)$. Since $V-V_{\infty}$ is $\Delta$-compact, it follows by the Weyl perturbation Theorem that the operator $P(h)$ admits a unique self-adjoint realization in $L^{2}\left(\mathbb{R}^{d} ; \mathbb{C}^{N}\right)$ with domain $H^{2}\left(\mathbb{R}^{d} ; \mathbb{C}^{N}\right)$ and for any fixed $h>0, \sigma_{\text {ess }}(P(h))=\sigma_{\text {ess }}\left(P_{\infty}(h)\right)=$ $\left[\lambda_{1},+\infty\right)$. Thus, the operator $P(h)$ may have discrete eigenvalues in $\left(-\infty, \lambda_{1}\right)$ and embedded ones in the interval $\left[\lambda_{1}, \lambda_{N}\right]$.

Let

$$
R_{h}(z):=(P(h)-z)^{-1} \in \mathcal{L}\left(L^{2}\left(\mathbb{R}^{d} ; \mathbb{C}^{N}\right)\right), \quad \Im z \neq 0,
$$

denotes the resolvent of $P(h)$. It follows by the Limiting Absorption Principle (see e.g. [Ag]), using the dilation generator as a scalar conjugate operator, that for any $E>\left\|V_{\infty}\right\|_{N \times N}$ and any $s>\frac{1}{2}$, the boundary value of the resolvent

$$
R_{h}(E \pm i 0):=\lim _{\varepsilon \rightarrow 0^{+}} R_{h}(E \pm i \varepsilon)
$$

exists as a bounded operator in $\mathcal{L}\left(L^{2, s}\left(\mathbb{R}^{d} ; \mathbb{C}^{N}\right), L^{2,-s}\left(\mathbb{R}^{d} ; \mathbb{C}^{N}\right)\right)$. Here $L^{2, s}\left(\mathbb{R}^{d} ; \mathbb{C}^{N}\right)$ denotes the space of $\mathbb{C}^{N}$-valued functions that are square integrable on $\mathbb{R}^{d}$ with respect to the measure $\langle x\rangle^{s} d x$, equipped with its natural norm

$$
\|u\|_{L^{2, s}\left(\mathbb{R}^{d} ; \mathbb{C}^{N}\right)}:=\left\|\langle x\rangle^{s} u\right\|_{L^{2}\left(\mathbb{R}^{d} ; \mathbb{C}^{N}\right)}=\left(\int_{\mathbb{R}^{d}}|u(x)|_{\mathbb{C}^{N}}^{2}\langle x\rangle^{2 s} d x\right)^{\frac{1}{2}} .
$$

A natural question is to study the behavior of $R_{h}(E \pm i 0)$ as $h \rightarrow 0^{+}$, more precisely, to estimate the size of the norm

$$
\left\|R_{h}(E \pm i 0)\right\|_{L^{2, s}\left(\mathbb{R}^{d} ; \mathbb{C}^{N}\right) \rightarrow L^{2,-s}\left(\mathbb{R}^{d} ; \mathbb{C}^{N}\right)}, \quad s>\frac{1}{2},
$$

with respect to $h$. This problem is important in scattering theory, for instance, for studying the behavior of observables like the scattering matrix and the total cross section (see e.g., [RT, Na, Mi]). Moreover, it is well known that the semiclassical behavior of the resolvent near a given energy-level have a deep relationship with the existence or the absence of resonance near this level. As we shall see later, getting estimates on the size of the above norm entails important results on the location of resonances of the operator $P(h)$ near the energy-level $E$. Let $\operatorname{Res}(P(h))$ denote the set of resonances of $P(h)$ which we will define rigourously in Section 2.

1.2. Background on the scalar case. In the scalar case $N=1$ (and $V_{\infty}=0$ ), it is well known that the size of $(1.3)$ is $\mathcal{O}\left(h^{-1}\right)$ in non-trapping situations, that is

$$
\left\|R_{h}(E \pm i 0)\right\|_{L^{2, s}\left(\mathbb{R}^{d}\right) \rightarrow L^{2,-s}\left(\mathbb{R}^{d}\right)} \lesssim h^{-1}, \quad \forall s>\frac{1}{2},
$$

provided that $E>0$ is non-trapping for the classical Hamiltonian $p(x, \xi):=\xi^{2}+V(x)$, $(x, \xi) \in T^{*} \mathbb{R}^{d}$, associated with $P(h)$. We recall that an energy $E>0$ is said to be nontrapping for $p$ if the set of trapped trajectories at $E$ defined by

$$
\mathcal{T}(E):=\left\{(x, \xi) \in p^{-1}(E) ; \exp \left(t H_{p}\right)(x, \xi) \nrightarrow \infty \text { as } t \rightarrow \pm \infty\right\}
$$

is empty. Here $\exp \left(t H_{p}\right): T^{*} \mathbb{R}^{d} \rightarrow T^{*} \mathbb{R}^{d}$ is the flow generated by the Hamiltonian vector field $H_{p}=2 \xi \cdot \partial_{x}-\nabla_{x} V \cdot \partial_{\xi}$. This result was proved by Robert and Tamura [RT] using Mourre theory and Fourier integral methods. In particular, estimate (1.4) is the key ingredient in the estimation of the behavior of the scattering cross-section and the complete asymptotic 
expansion in powers of $h$ for the spectral shift function associated to the operator pair $\left(-h^{2} \Delta+\right.$ $\left.V(x),-h^{2} \Delta\right)$. A shorter proof using the construction of a global escape function and Mourre theory was given later by Gérard and Martinez [GM].

Another consequence of the non-trapping hypothesis on the energy $E>0$ is the following absence of resonances result due to Martinez [Ma]

$$
\operatorname{Res}(P(h)) \cap\left\{z \in \mathbb{C} ; \operatorname{Re} z \in\left[E-\varepsilon_{0}, E+\varepsilon_{0}\right] \text { and } \operatorname{Im} z \geq-C h|\ln h|\right\}=\emptyset,
$$

for some $\varepsilon_{0}>0$ and all $C>0, h \in\left(0, h_{C}\right]$. Martinez's approach is based on some microlocal weighted estimates combined with the construction of a global escape function associated to the classical Hamiltonian $p$. An alternative approach to conjugated operators was introduced later by Sjöstrand and Zworski [SZ2].

Now, without any assumption on the set of trapped trajectories (trapping situations), we have the following estimates for any $s>\frac{1}{2}$ and $E>0$,

$$
\begin{gathered}
\left\|R_{h}(E \pm i 0)\right\|_{L^{2, s}\left(\mathbb{R}^{d}\right) \rightarrow L^{2,-s}\left(\mathbb{R}^{d}\right)} \lesssim e^{\frac{C}{h}}, \\
\left\|\mathbb{1}_{\left\{x \in \mathbb{R}^{d} ;|x| \geq R_{0}\right\}} R_{h}(E \pm i 0) \mathbb{1}_{\left\{x \in \mathbb{R}^{d} ;|x| \geq R_{0}\right\}}\right\|_{L^{2, s}\left(\mathbb{R}^{d}\right) \rightarrow L^{2,-s}\left(\mathbb{R}^{d}\right)} \lesssim h^{-1},
\end{gathered}
$$

for some constants $C, R_{0}>0$ and $h>0$ small enough. Here $\mathbb{1}_{\left\{x \in \mathbb{R}^{d} ;|x| \geq R_{0}\right\}}$ is the characteristic function of the set $\left\{x \in \mathbb{R}^{d} ;|x| \geq R_{0}\right\}$. These estimates are originally due to Burq [Bu] in a more general framework including perturbations of second-order elliptic operators with smooth coefficients, obstacle scattering and metric scattering. Others proofs was given later by Vodev [Vol] and Sjöstrand [Sj]. Cardoso and Vodev [CV] and recently Rodianski and Tao [RoTa] established generalizations of these estimates to Schrödinger operators on manifolds. Carleman estimates are the main tool in all these works. Recently, Datchev [Da] provided an elementary proof of these estimates for scalar Schrödinger operators on $\mathbb{R}^{d}, d \neq 2$ (see [Sh] for the case $d=2$ ). The proof in [Da] is based on a global weighted Carleman estimate which has the advantage to be valid under low regularity assumption on the potential $V$ and where the construction of the weight function is simple and explicit. More precisely, Datchev's method only requires that the potential $V$ is bounded on $\mathbb{R}^{d}$ together with its radial derivative, and they decay like $(1+|x|)^{-\rho_{0}}$ and $(1+|x|)^{-\rho_{0}-1}$ at infinity, respectively.

The $h$-dependence in (1.5) and (1.6) is optimal in general, that is without any assumption on the underlying classical dynamics. Moreover, estimate (1.6) is not true in general when removing one of the characteristic function $\mathbb{1}_{\left\{x \in \mathbb{R}^{d} ;|x| \geq R_{0}\right\}}$ (see [DDZ]).

As a consequence of estimate (1.5), Burq [Bu] proved the following resonance free region

$$
\operatorname{Res}(P(h)) \cap\left\{z \in \mathbb{C} ; \operatorname{Re} z \in J \text { and } \operatorname{Im} z \geq-C e^{-C / h}\right\}=\emptyset,
$$

for any compact interval $J \subset(0,+\infty)$ and some constant $C>0$, and $h>0$ small enough.

1.3. Comments on the matrix-valued case. In the matrix-valued case, that is, when the considered operator is of the form (1.1), the situation is more complicated. Notice that since the eigenvalues are not enough regular in general, the usual definition of the Hamiltonian flow for a matrix-valued Hamiltonian function does not make sense (see [Ka]). The nontrapping condition in this case is usually characterized by the existence of a global escape 
function associated with the matrix-valued symbol of the operator $P(h)$ at the considered energy-level.

Let $p(x, \xi):=|\xi|^{2} I_{N}+V(x),(x, \xi) \in T^{*} \mathbb{R}^{d}$, be the matrix-valued semiclassical symbol of $P(h)$. We denote $\lambda_{1}(x) \leq \lambda_{2}(x) \leq \cdots \leq \lambda_{N}(x)$ the (real) eigenvalues of $V(x), x \in \mathbb{R}^{d}$. In general, the functions $x \mapsto \lambda_{j}(x)$ are continuous on $\mathbb{R}^{d}, j=1, \ldots, N$. For an energy $E \in \mathbb{R}$, we denote $\Sigma_{E}$ the corresponding energy surface defined by

$$
\Sigma_{E}:=\bigcup_{j=1}^{N}\left\{(x, \xi) \in T^{*} \mathbb{R}^{d} ;|\xi|^{2}+\lambda_{j}(x)=E\right\} .
$$

A smooth real-valued function $G \in C^{\infty}\left(T^{*} \mathbb{R}^{d} ; \mathbb{R}\right)$ is a global escape function associated with the classical Hamiltonian $p$ at $E$ if there exists a constant $c>0$ such that $\{p, G\}_{\mid \Sigma_{E}} \geq c I_{N}$ in the sense of hermitian matrices, i.e.,

$$
(\{p, G\}(x, \xi) w, w)_{\mathbb{C}^{N}} \geq c|w|^{2}, \quad \forall(x, \xi) \in \Sigma_{E}, \forall w \in \mathbb{C}^{N}
$$

Here $\{p, G\}:=\partial_{\xi} p \cdot \partial_{x} G-\partial_{x} p \cdot \partial_{\xi} G$ denotes the Poisson bracket of $p$ and $G$, and $(\cdot, \cdot)_{\mathbb{C}^{N}}$ denotes the inner product in $\mathbb{C}^{N}$.

In the scalar case $N=1$, the existence of a global escape function associated with the symbol $p$ at an energy $E>0$ is equivalent to the non-trapping condition (see for instance [GM]). In [Je3], under the existence condition of a global escape function, Jecko proved that estimate (1.4) still holds in the case of matrix-valued potentials. With this result at hand, the main challenge consists in the construction of a global escape function which may be quite complicated. This question has been the subject of many works (see [Je2, Je3, Je4, FR] and the references therein) for different type of eigenvalue crossings.

In this note, we provide generalizations of Burq's estimates (1.5) and (1.6) to the case of Schrödinger operators with matrix-valued potentials using the approach developed in [Da] and we prove related results on the absence of resonances near the real axis. We refer to [As] for applications of these results to scattering theory. We also refer to ([Ash, FMW1, FMW2, FMW3, Hi] and the references therein) for some recent works on the widths of resonances for systems of coupled Schrödinger operators for different potentials and at different energy levels.

\section{Statement of the Results}

Let $\mathcal{H}_{N}$ denotes the space of $N \times N$ hermitian matrices endowed with the norm $\|\cdot\|_{N \times N}$, where for $\mathcal{M} \in \mathcal{H}_{N}$,

$$
\|\mathcal{M}\|_{N \times N}:=\sup _{\left\{w \in \mathbb{R}^{N} ;|w| \leq 1\right\}}|\mathcal{M} w|
$$

2.1. Resolvent estimates. Consider the semiclassical Schrödinger operator on $L^{2}\left(\mathbb{R}^{d} ; \mathbb{C}^{N}\right)$, $d \neq 2$,

$$
P(h):=-h^{2} \Delta \cdot I_{N}+V(x),
$$

where $I_{N}$ is the identity $N \times N$ matrix and $h>0$ is the semiclassical parameter. Using the polar coordinates $\mathbb{R}^{d} \ni x=(r, \omega) \in \mathbb{R}_{+} \times \mathbb{S}^{d-1}$, where $\mathbb{S}^{d-1}$ denotes the unit sphere on $\mathbb{R}^{d}$, we assume the following conditions on the potential $V$. 
Assumption (A1). $V: \mathbb{R}_{+} \times \mathbb{S}^{d-1} \rightarrow \mathcal{H}_{N}$ and its distributional derivative $\partial_{r} V$ are bounded on $\mathbb{R}_{+} \times \mathbb{S}^{d-1}$, i.e.,

$$
V, \partial_{r} V \in L^{\infty}\left(\mathbb{R}_{+} \times \mathbb{S}^{d-1} ; \mathcal{H}_{N}\right),
$$

and has the following long-range behavior at infinity:

Assumption (A2). There exist a constant hermitian matrix $V_{\infty} \in \mathcal{H}_{N}$ and $\rho_{0}>0$ such that

$$
\left\|V(r, \omega)-V_{\infty}\right\|_{N \times N} \leq(1+r)^{-\rho_{0}}, \quad\left\|\partial_{r} V(r, \omega)\right\|_{N \times N} \leq(1+r)^{-\rho_{0}-1},
$$

for all $(r, \omega) \in \mathbb{R}_{+} \times \mathbb{S}^{d-1}$.

We have the following resolvent estimates.

Theorem 2.1. Assume (A1) and (A2). For any $E>\left\|V_{\infty}\right\|_{N \times N}$ and $s>\frac{1}{2}$, there exist $C, R_{0}, h_{0}>0$ such that for all $h \in\left(0, h_{0}\right]$ and $\varepsilon>0$, the following estimates hold

$$
\begin{gathered}
\left\|R_{h}(E \pm i \varepsilon)\right\|_{L^{2, s}\left(\mathbb{R}^{d} ; \mathbb{C}^{N}\right) \rightarrow L^{2,-s}\left(\mathbb{R}^{d} ; \mathbb{C}^{N}\right)} \leq e^{\frac{C}{h}}, \\
\left\|\mathbb{1}_{\left\{x \in \mathbb{R}^{d} ;|x| \geq R_{0}\right\}} R_{h}(E \pm i \varepsilon) \mathbb{1}_{\left\{x \in \mathbb{R}^{d} ;|x| \geq R_{0}\right\}}\right\|_{L^{2, s}\left(\mathbb{R}^{d} ; \mathbb{C}^{N}\right) \rightarrow L^{2,-s}\left(\mathbb{R}^{d} ; \mathbb{C}^{N}\right)} \leq C h^{-1} .
\end{gathered}
$$

Here $\mathbb{1}_{\left\{x \in \mathbb{R}^{d} ;|x| \geq R_{0}\right\}}$ is the characteristic function of the set $\left\{x \in \mathbb{R}^{d} ;|x| \geq R_{0}\right\}$.

Remark 2.2. Estimates on resolvent truncated outside a large compact set are important in scattering theory. Indeed, the operator $\mathbb{1}_{\left\{x \in \mathbb{R}^{d} ;|x| \geq R_{0}\right\}} R_{h}(z) \mathbb{1}_{\left\{x \in \mathbb{R}^{d} ;|x| \geq R_{0}\right\}}$ appears for instance in the representation of the scattering amplitude for compactly supported perturbations (see [PZ]). In [As], we apply (2.5) to prove estimates on the scattering amplitude for Schrödinger operators with matrix-valued potentials.

2.2. Resonance free domains. Now, we state our results on the resonances of $P(h)$. To define the resonances of $P(h)$, we need the following assumption on the potential $V$.

Assumption $\left(\mathbf{H o l}_{\infty}\right) . V \in C^{\infty}\left(\mathbb{R}^{d} ; \mathcal{H}_{N}\right)$ and extends to an analytic function on $\mathcal{S} \subset \mathbb{C}^{d}$

$$
\mathcal{S}:=\left\{x \in \mathbb{C}^{d} ;|\operatorname{Im} x| \leq c_{0}\langle\operatorname{Re} x\rangle,|\operatorname{Re} x|>\kappa\right\},
$$

for some constants $c_{0}, \kappa>0$. Moreover, there exist $\rho_{0}>0$ and a constant $C>0$ such that for all $x \in \mathcal{S}$

$$
\left\|V(x)-V_{\infty}\right\|_{N \times N} \leq C\langle x\rangle^{-\rho_{0}}, \quad\langle x\rangle:=\left(1+|x|^{2}\right)^{\frac{1}{2}} .
$$

Under this assumption, we can define the resonances of $P(h)$ near the real axis by the method of complex distortion as it was done in [Hu, Ne] (see also [AC, SZ1] for an alternative approach). Let $A \gg 1$ be a large constant, and let $F: \mathbb{R}^{d} \rightarrow \mathbb{R}^{d}$ be a smooth vector-field such that

$$
F(x)=\left\{\begin{array}{l}
0 \text { for }|x| \leq A \\
x \text { for }|x| \geq A+1
\end{array}\right.
$$

We introduce the one-parameter family of unitary distortion

$$
C_{0}^{\infty}\left(\mathbb{R}^{d} ; \mathbb{C}^{N}\right) \ni f \longmapsto U_{\omega} f(x):=\left|J_{\phi_{\omega}(x)}\right|^{\frac{1}{2}} f\left(\phi_{\omega}(x)\right), \quad \omega \in \mathbb{R},
$$

where $\phi_{\omega}(x):=x+\omega F(x)$ and $J_{\phi_{\omega}(x)}:=\operatorname{det}(1+\omega \nabla F(x))$ is the Jacobian of $\phi_{\omega}(x)$.

For $\omega \in \mathbb{R}$ small enough, $U_{\omega}$ extends to a unitary operator on $L^{2}\left(\mathbb{R}^{d} ; \mathbb{C}^{N}\right)$. We define

$$
P_{\omega}(h):=U_{\omega} P(h)\left(U_{\omega}\right)^{-1} .
$$


Under the assumption $\left(\mathbf{H o l}_{\infty}\right)$ on the potential $V$, the operator $P_{\omega}(h)$ is a differential operator with analytic coefficients with respect to $\omega$, and can therefore be continued in a unique way to small enough complex values of $\omega$. Therefore, the distorted operator

$$
P_{\theta}(h):=U_{i \theta} P(h)\left(U_{i \theta}\right)^{-1}
$$

is well defined for $\theta>0$ small enough. By Weyl perturbation theorem its essential spectrum is given by

$$
\sigma_{\text {ess }}\left(P_{\theta}(h)\right)=\bigcup_{j=1}^{N}\left(\lambda_{j}+e^{-2 i \theta} \mathbb{R}_{+}\right) .
$$

Hence, the spectrum of $P_{\theta}(h)$ in the complex sector

$$
S_{\theta}:=\left(\lambda_{1}+e^{-2 i[0, \theta)} \mathbb{R}_{+}^{*}\right) \backslash\left(\cup_{j=1}^{N}\left(\lambda_{j}+e^{-2 i \theta} \mathbb{R}_{+}\right)\right)
$$

is discrete, consists on isolated eigenvalues with finite multiplicities. Moreover, standard arguments (see e.g. [HM]) show that $\sigma\left(P_{\theta}(h)\right) \cap S_{\theta}$ does not depends on the particular choice of the vector-field $F$ and for any $0<\theta<\theta^{\prime}$ small enough and $h>0$ fixed, we have

$$
\sigma\left(P_{\theta}(h)\right) \cap S_{\theta}=\sigma\left(P_{\theta^{\prime}}(h)\right) \cap S_{\theta} .
$$

The resonances of $P(h)$ in $S_{\theta}$ are defined as the eigenvalues of $P_{\theta}(h)$ in $S_{\theta}$, or equivalently as the eigenvalues of $P_{\theta^{\prime}}(h)$ in $S_{\theta}$ for all $0<\theta<\theta^{\prime}$ small enough. In the following, we denote $\operatorname{Res}(P(h))$ the set of resonances of $P(h)$.

The estimate (2.4) entails the following result on the absence of resonances in an exponentially small band below $\left(\left\|V_{\infty}\right\|_{N \times N},+\infty\right)$.

Theorem 2.3. Assume $\left(\mathbf{H o l}_{\infty}\right)$. For any compact interval $J \subset\left(\left\|V_{\infty}\right\|_{N \times N},+\infty\right)$, there exist a constant $C>0$ and $h_{0} \in(0,1]$ such that for all $h \in\left(0, h_{0}\right]$, we have

$$
\operatorname{Res}(P(h)) \cap\left\{z \in \mathbb{C} ; \operatorname{Re} z \in J \text { and } \operatorname{Im} z \geq-C e^{-C / h}\right\}=\emptyset .
$$

Remark 2.4. Notice that assumption $\left(\mathbf{H o l}_{\infty}\right)$ and the Cauchy formula imply assumptions (A1) and (A2).

In our proof of Theorem 2.3, we shall use the following result which generalizes the wellknown result of $[\mathrm{Ma}]$ to the case of Schrödinger operators with matrix-valued potentials.

Theorem 2.5. Assume $\left(\mathbf{H o l}_{\infty}\right)$. Let $E_{0}>\left\|V_{\infty}\right\|_{N \times N}$ and suppose that there exists $G \in$ $C^{\infty}\left(T^{*} \mathbb{R}^{d} ; \mathbb{R}\right)$ such that $(1.7)$ holds on $\Sigma_{E_{0}}$. Then there exists $\varepsilon_{0}>0$ such that for all $C>0$, there exists $h_{C} \in(0,1]$ such that for all $0<h \leq h_{C}$, we have

$$
\operatorname{Res}(P(h)) \cap\left\{z \in \mathbb{C} ; \operatorname{Re} z \in\left[E_{0}-\varepsilon_{0}, E_{0}+\varepsilon_{0}\right] \text { and } \operatorname{Im} z \geq-C h|\ln h|\right\}=\emptyset .
$$

Remark 2.6. In the scalar case $N=1$, a quantitative version of the previous result in terms of an estimate on the distorted resolvent was proved in [NSZ] (see also [SZ2]). More precisely, if $P_{\theta}(h)$ denotes the distorted operator with $\theta=\theta(h)=C^{\prime} h|\ln h|, C^{\prime} \gg C$, then for any $C>0$ there exist a constant $C^{\prime \prime}>0$ and $h_{C}>0$ such that

$$
\left\|\left(P_{\theta}(h)-z\right)^{-1}\right\| \leq \frac{C^{\prime \prime}}{h} \exp \left(C^{\prime \prime}|\operatorname{Im} z| / h\right),
$$

uniformly for $z \in\left\{z \in \mathbb{C}\right.$; $\operatorname{Re} z \in\left[E_{0}-\varepsilon_{0}, E_{0}+\varepsilon_{0}\right]$ and $\left.\operatorname{Im} z \geq-C h|\ln h|\right\}$ and $h \in\left(0, h_{C}\right]$. 
In Lemma 4.1, we prove a weaker estimate (see (4.2)) on the distorted resolvent which coincides with (2.11) for $\operatorname{Im} z=C h|\ln h|$. As in [NSZ], using a version of the non-trapping estimate and the semiclassical maximum principle, we can prove the same estimate (2.11) for our matrix-valued operator. This will be proved in details in [As].

\section{Resolvent estimates}

In this section, we present the main ideas of the proof of Theorem 2.1 referring to [As] for the details. We follow the approach developed in [Da] which we adapt in our context of matrix-valued operator. The main step in the proof of estimates (2.4) and (2.5) is the following global weighted Carleman estimate. In the following $s>\frac{1}{2}$ and $E>\left\|V_{\infty}\right\|_{N \times N}$ are fixed.

Proposition 3.1. There exist $R_{0}, h_{0}, C>0$ and a positive radial function $\varphi=\varphi(r) \in$ $C^{\infty}\left(\mathbb{R}_{+} ; \mathbb{R}_{+}\right)$with $\varphi^{\prime} \geq 0$ and $\operatorname{supp} \varphi^{\prime}=\left[0, R_{0}\right]$ such that the following estimate holds

$$
h^{2}\left\|e^{\varphi / h} v\right\|_{L^{2,-s}\left(\mathbb{R}^{d} ; \mathbb{C}^{N}\right)}^{2} \leq C\left\|e^{\varphi / h}\left(P(h)-(E+i \varepsilon) I_{N}\right) v\right\|_{L^{2, s}\left(\mathbb{R}^{d} ; \mathbb{C}^{N}\right)}^{2}+C \varepsilon h\left\|e^{\varphi / h} v\right\|_{L^{2}\left(\mathbb{R}^{d} ; \mathbb{C}^{N}\right)}^{2},
$$

for all $\varepsilon \geq 0, h \in\left(0, h_{0}\right]$ and $v \in C_{0}^{\infty}\left(\mathbb{R}^{d} ; \mathbb{C}^{N}\right)$.

The proof of this result relies mainly on two steps.

Step 1. The first step consists in the construction of a weight function $\varphi \in C^{\infty}\left(\mathbb{R}_{+} ; \mathbb{R}_{+}\right)$ such that for some $0<R<R_{0}, \varphi$ increases linearly on $[0, R], \varphi$ increases slowly on $\left[R, R_{0}\right]$ and $\varphi=$ Cste on $\left[R_{0},+\infty\right)$ (see Figure 1 ), so that it satisfies a nice estimate in relation with the potential $V$, more precisely,

$$
\left(\frac{1}{m^{\prime}(r)} \partial_{r}\left[m(r)\left(E I_{N}-V_{\varphi}(r, \omega ; h)\right)\right] w, w\right)_{\mathbb{C}^{N}} \geq C|w|_{\mathbb{C}^{N}}^{2}
$$

for some constant $C>0$, uniformly with respect to $(r, \omega) \in(0,+\infty) \times \mathbb{S}^{d-1}, h \in\left(0, h_{0}\right]$ and $w \in \mathbb{C}^{N}$, where $m(r):=1-(1+r)^{1-2 s}$ and

$$
V_{\varphi}(r, \omega ; h):=V(r, \omega)-\left(\left(\varphi^{\prime}(r)\right)^{2}-h \varphi^{\prime \prime}(r)\right) I_{N} .
$$

Here $(\cdot, \cdot)_{\mathbb{C}^{N}}$ and $|\cdot|_{\mathbb{C}^{N}}$ denote the hermitian inner product and norm in $\mathbb{C}^{N}$.

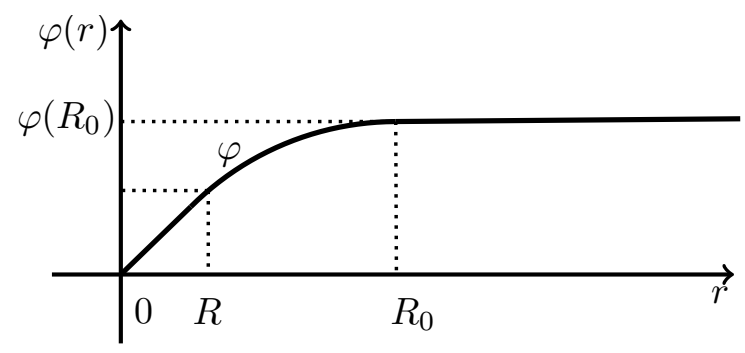

Figure 1. The weight function $\varphi$ 
Step 2. Now, using the weighted function $\varphi$ constructed above, we introduce the conjugated operator

$$
\mathcal{P}_{\varphi}=\mathcal{P}_{\varphi}(h):=e^{\varphi / h} r^{(d-1) / 2}\left(P(h)-(E+i \varepsilon) I_{N}\right) r^{-(d-1) / 2} e^{-\varphi / h} .
$$

We have

$$
\mathcal{P}_{\varphi}=\left(-h^{2} \partial_{r}^{2}+2 h \varphi^{\prime}(r) \partial_{r}+\mathcal{Q}\right) \cdot I_{N}+V_{\varphi}(r, \omega ; h)-(E+i \varepsilon) I_{N},
$$

where $V_{\varphi}$ is the effective potential defined by (3.3) and

$$
\mathcal{Q}=\mathcal{Q}(h):= \begin{cases}0 & (d=1) \\ h^{2} r^{-2}\left(-\Delta_{\mathbb{S}^{d-1}}+\frac{(d-1)(d-3)}{4}\right) & (d \geq 3) .\end{cases}
$$

Here $\Delta_{\mathbb{S}^{d-1}}$ denotes the Laplacian on the unit sphere $\mathbb{S}^{d-1}$.

Passing to polar coordinates using that $L^{2}\left(\mathbb{R}^{d}, d x\right)=L^{2}\left(\mathbb{R}_{+} \times \mathbb{S}^{d-1}, r^{d-1} d r d \omega\right)$, the estimate (3.1) is equivalent to the following one

$$
\iint_{r, \omega} m^{\prime}(r)|u(r, \omega)|_{\mathbb{C}^{N}}^{2} d r d \omega \leq \frac{C}{h^{2}} \iint_{r, \omega} \frac{\left|\mathcal{P}_{\varphi} u(r, \omega)\right|_{\mathbb{C}^{N}}^{2}}{m^{\prime}(r)} d r d \omega+\frac{C \varepsilon}{h} \iint_{r, \omega}|u(r, \omega)|_{\mathbb{C}^{N}}^{2} d r d \omega
$$

for all $u \in e^{\varphi / h} r^{(d-1) / 2} C_{0}^{\infty}\left(\mathbb{R}^{d} ; \mathbb{C}^{N}\right)$.

Let $\langle\cdot, \cdot\rangle_{\mathbb{S}^{d-1}}$ and $\|\cdot\|_{\mathbb{S}^{d-1}}$ denote the inner product and norm in $L^{2}\left(\mathbb{S}^{d-1} ; \mathbb{C}^{N}\right)$, and we introduce the functional

$$
\mathcal{L}_{h}(r):=\left\|h u^{\prime}(r, \omega)\right\|_{\mathbb{S}^{d-1}}^{2}-\left\langle\left(\mathcal{Q} \cdot I_{N}+V_{\varphi}(r, \omega ; h)-E I_{N}\right) u, u\right\rangle_{\mathbb{S}^{d-1}}, \quad r>0,
$$

where here and in the sequel prime notation always denote differentiation with respect to $r$, for instance $u^{\prime}:=\partial_{r} u$. Using the self-adjointness of

$$
\mathcal{Q} \cdot I_{N}+V_{\varphi}-E I_{N}=\mathcal{P}_{\varphi}-\left(-h^{2} \partial_{r}^{2}+2 h \varphi^{\prime}(r) \partial_{r}-i \varepsilon\right) \cdot I_{N}
$$

we get

$$
\begin{aligned}
\mathcal{L}_{h}^{\prime}(r)= & -2 \operatorname{Re}\left\langle\mathcal{P}_{\varphi} u, u^{\prime}\right\rangle_{\mathbb{S}^{d-1}}+4 h \varphi^{\prime}(r)\left\|u^{\prime}\right\|_{\mathbb{S}^{d-1}}^{2}+2 \varepsilon \operatorname{Im}\left\langle u, u^{\prime}\right\rangle_{\mathbb{S}^{d-1}} \\
& +2 r^{-1}\left\langle\mathcal{Q} \cdot I_{N} u, u\right\rangle_{\mathbb{S}^{d-1}}-\left\langle V_{\varphi}^{\prime} u, u\right\rangle_{\mathbb{S}^{d-1}} .
\end{aligned}
$$

It follows that

$$
\begin{aligned}
\left(m \mathcal{L}_{h}\right)^{\prime}(r)= & -2 m \operatorname{Re}\left\langle\mathcal{P}_{\varphi} u, u^{\prime}\right\rangle_{\mathbb{S}^{d-1}}+\left(4 h^{-1} m \varphi^{\prime}+m^{\prime}\right)\left\|h u^{\prime}\right\|_{\mathbb{S}^{d-1}}^{2}+2 m \varepsilon \operatorname{Im}\left\langle u, u^{\prime}\right\rangle_{\mathbb{S}^{d-1}} \\
& +\left(2 m r^{-1}-m^{\prime}\right)\left\langle\mathcal{Q} \cdot I_{N} u, u\right\rangle_{\mathbb{S}^{d-1}}+\left\langle\partial_{r}\left[m\left(E I_{N}-V_{\varphi}\right)\right] u, u\right\rangle_{\mathbb{S}^{d-1}} .
\end{aligned}
$$

First, using the fact that

$$
m \varphi^{\prime} \geq 0, m^{\prime}>0, \quad \mathcal{Q} \geq 0,2 m r^{-1}-m^{\prime}>0,
$$

we obtain

$$
\begin{aligned}
\left(m \mathcal{L}_{h}\right)^{\prime}(r) \geq & -2 m \operatorname{Re}\left\langle\mathcal{P}_{\varphi} u, u^{\prime}\right\rangle_{\mathbb{S}^{d-1}}+m^{\prime}\left\|h u^{\prime}\right\|_{\mathbb{S}^{d-1}}^{2}+\left\langle\partial_{r}\left[m\left(E I_{N}-V_{\varphi}\right)\right] u, u\right\rangle_{\mathbb{S}^{d-1}} \\
& +2 m \varepsilon \operatorname{Im}\left\langle u, u^{\prime}\right\rangle_{\mathbb{S}^{d-1}} .
\end{aligned}
$$

Then, using the inequality $-2 \operatorname{Re}\langle a, b\rangle+\|b\|^{2} \geq-\|a\|^{2}$, we get

$$
\left(m \mathcal{L}_{h}\right)^{\prime}(r) \geq-\frac{1}{h^{2} m^{\prime}}\left\|\mathcal{P}_{\varphi} u\right\|_{\mathbb{S}^{d-1}}^{2}+2 m \varepsilon \operatorname{Im}\left\langle u, u^{\prime}\right\rangle_{\mathbb{S}^{d-1}}+\left\langle\partial_{r}\left[m\left(E I_{N}-V_{\varphi}\right)\right] u, u\right\rangle_{\mathbb{S}^{d-1}}
$$


By integrating with respect to $r$ using the fact that $\int_{0}^{+\infty}\left(m \mathcal{L}_{h}\right)^{\prime}(r) d r=0$ and the CauchySchwarz inequality, we obtain

$$
\iint_{r, \omega}\left(\partial_{r}\left[m\left(E I_{N}-V_{\varphi}\right)\right] u, u\right)_{\mathbb{C}^{N}} d r d \omega \leq \frac{1}{h^{2}} \iint_{r, \omega} \frac{\left|\mathcal{P}_{\varphi} u\right|_{\mathbb{C}^{N}}^{2}}{m^{\prime}(r)} d r d \omega+2 \varepsilon \iint_{r, \omega}|u|_{\mathbb{C}^{N}}\left|u^{\prime}\right|_{\mathbb{C}^{N}} d r d \omega .
$$

It follows from estimate (3.2) that

$$
C \iint_{r, \omega} m^{\prime}(r)|w|_{\mathbb{C}^{N}}^{2} d r d \omega \leq \frac{1}{h^{2}} \iint_{r, \omega} \frac{\left|\mathcal{P}_{\varphi} u\right|_{\mathbb{C}^{N}}^{2}}{m^{\prime}(r)} d r d \omega+2 \varepsilon \iint_{r, \omega}|u|_{\mathbb{C}^{N}}\left|u^{\prime}\right|_{\mathbb{C}^{N}} d r d \omega,
$$

for some constant $C>0$. On the other hand, it is not difficult to prove that there exists a constant $C^{\prime}>0$ such that

$$
2 \varepsilon \iint_{r, \omega}|u|_{\mathbb{C}^{N}}\left|u^{\prime}\right|_{\mathbb{C}^{N}} d r d \omega \leq \frac{C^{\prime} \varepsilon}{h} \iint_{r, \omega}|u|_{\mathbb{C}^{N}}^{2} d r d \omega+\frac{C^{\prime} \varepsilon}{h} \iint_{r, \omega}\left|\mathcal{P}_{\varphi} u\right|_{\mathbb{C}^{N}}^{2} d r d \omega .
$$

Putting together (3.5) and (3.6) we obtain (3.4). This ends the proof of Proposition 3.1.

Now, using the fact that $\varphi(r)=\frac{C_{0}}{2}$ for $r \geq R_{0}$, with $C_{0}=2 \max _{(0,+\infty)} \varphi$, and estimate (3.1), we obtain

$$
\begin{aligned}
e^{-C_{0} / h}\left\|\mathbb{1}_{\left\{x \in \mathbb{R}^{d} ;|x| \leq R_{0}\right\}} v\right\|_{L^{2,-s}}^{2}+\left\|\mathbb{1}_{\left\{x \in \mathbb{R}^{d} ;|x| \geq R_{0}\right\}} v\right\|_{L^{2,-s}}^{2} \leq \\
\frac{C}{h^{2}}\left\|\left(P-(E+i \varepsilon) I_{N}\right) v\right\|_{L^{2, s}}^{2}+\frac{C \varepsilon}{h}\|v\|_{L^{2}}^{2},
\end{aligned}
$$

for some constant $C>0$, uniformly for $v \in C_{0}^{\infty}\left(\mathbb{R}^{d} ; \mathbb{C}^{N}\right), \varepsilon \geq 0$ and $h>0$ small enough. On the other hand, using the selfadjointness of $P$ and the Cauchy-Schwarz inequality, we get, for any $\varepsilon \geq 0, C^{\prime}>0$ and $h>0$ small enough,

$$
\begin{aligned}
2 \varepsilon\|v\|_{L^{2}}^{2} & =-2 \operatorname{Im}\left\langle\left(P-(E+i \varepsilon) I_{N}\right) v, v\right\rangle_{L^{2}} \\
& \leq \frac{C^{\prime}}{h}\left\|\mathbb{1}_{\left\{x \in \mathbb{R}^{d} ;|x| \geq R_{0}\right\}}\left(P-(E+i \varepsilon) I_{N}\right) v\right\|_{L^{2, s}}^{2}+\frac{h}{C^{\prime}}\left\|\mathbb{1}_{\left\{x \in \mathbb{R}^{d} ;|x| \geq R_{0}\right\}} v\right\|_{L^{2,-s}}^{2} \\
& +e^{2 C_{0} / h}\left\|\mathbb{1}_{\left\{x \in \mathbb{R}^{d} ;|x| \leq R_{0}\right\}}\left(P-(E+i \varepsilon) I_{N}\right) v\right\|_{L^{2, s}}^{2}+e^{-2 C_{0} / h}\left\|\mathbb{1}_{\left\{x \in \mathbb{R}^{d} ;|x| \leq R_{0}\right\}} v\right\|_{L^{2,-s}}^{2} .
\end{aligned}
$$

Combining (3.7) and (3.8), we get

$$
\begin{aligned}
& e^{-C / h}\left\|\mathbb{1}_{\left\{x \in \mathbb{R}^{d} ;|x| \leq R_{0}\right\}} v\right\|_{L^{2,-s}}^{2}+\left\|\mathbb{1}_{\left\{x \in \mathbb{R}^{d} ;|x| \geq R_{0}\right\}} v\right\|_{L^{2,-s}}^{2} \leq \\
& \quad e^{C / h}\left\|\mathbb{1}_{\left\{x \in \mathbb{R}^{d}|x| \leq R_{0}\right\}}\left(P-(E+i \varepsilon) I_{N}\right) v\right\|_{L^{2, s}}^{2}+\frac{C}{h^{2}}\left\|\mathbb{1}_{\left\{x \in \mathbb{R}^{d} ;|x| \geq R_{0}\right\}}\left(P-(E+i \varepsilon) I_{N}\right) v\right\|_{L^{2, s}}^{2},
\end{aligned}
$$

uniformly for $v \in C_{0}^{\infty}\left(\mathbb{R}^{d} ; \mathbb{C}^{N}\right), \varepsilon \geq 0$ and $h>0$ small enough.

Using this estimate, the proof can be finished by the density argument of [Da].

\section{RESONANCE FREE DOMAINS}

This section is devoted to the proofs of Theorems 2.3 and 2.5. We use the ordinary notations and some basic results of semiclassical analysis referring for example to the textbooks [DiSj, $\mathrm{Zw}$ ] for a clear presentation of this theory.

We first prove Theorem 2.5 using the approach to conjugate operators developed in [SZ2]. 
4.1. Proof of Theorem 2.5. Fix $E_{0}>\left\|V_{\infty}\right\|_{N \times N}$, and let

$$
p(x, \xi):=|\xi|^{2} I_{N}+V(x), \quad(x, \xi) \in T^{*} \mathbb{R}^{d},
$$

be the semiclassical symbol of $P(h)$. Let $G \in C^{\infty}\left(T^{*} \mathbb{R}^{d} ; \mathbb{R}\right)$ be an escape function associated with $p$ at $E_{0}$, i.e., $G$ satisfies $(1.7)$ on $\Sigma_{E_{0}}$. For $|(x, \xi)|$ large enough, the function $T^{*} \mathbb{R}^{d} \ni$ $(x, \xi) \mapsto x \cdot \xi$ is an escape function associated with $p$ at $E_{0}$. Indeed, we have

$$
\{p, x \cdot \xi\}(x, \xi)=2|\xi|^{2} I_{N}-x \cdot \nabla_{x} V(x), \quad(x, \xi) \in T^{*} \mathbb{R}^{d} .
$$

The assumption $\left(\mathbf{H o l}_{\infty}\right)$ and the Cauchy formula imply that $x \cdot \nabla_{x} V(x) \rightarrow 0$ as $|x| \rightarrow+\infty$. On the other hand, by the assumption $\left(\mathbf{H o l}_{\infty}\right),|\xi|^{2} \geq\left(E_{0}-\left\|V_{\infty}\right\|_{N \times N}\right) / 2$ on the energy surface $\Sigma_{E_{0}}$ for $|(x, \xi)|$ large enough. Therefore,

$$
\{p, x \cdot \xi\}(x, \xi) \geq\left(E_{0}-\left\|V_{\infty}\right\|_{N \times N}\right) / 2,
$$

for all $(x, \xi) \in \Sigma_{E_{0}} \cap\{|(x, \xi)| \gg 1\}$. Thus, without any loss of generality, we may assume that $G(x, \xi)=x \cdot \xi$ for $|(x, \xi)|$ large enough.

We set

$$
\mathcal{G}(x, \xi):=G(x, \xi)-F(x) \cdot \xi \in C_{0}^{\infty}\left(\mathbb{R}^{d} ; \mathbb{R}\right)
$$

where $F$ is the vector field used in the complex distortion (see $(2.8)$ ). Let $\mathcal{G}_{h}:=\mathrm{Op}_{h}^{w}(\mathcal{G})$ be the pseudodifferential operator with symbol $\mathcal{G}$.

Let $M>0$ be independent of $h$ and set $\theta_{1}=\theta_{1}(h)=i M \kappa(h)$ with $\kappa(h):=h|\ln h|$. We introduce the conjugated operator

$$
\widetilde{P}_{\theta_{1}}(h):=e^{-\frac{M \kappa(h)}{h} \mathcal{G}_{h}} P_{\theta_{1}}(h) e^{\frac{M \kappa(h)}{h} \mathcal{G}_{h}} .
$$

Since $\mathcal{G}$ is compactly supported it follows by the Calderón-Vaillancourt theorem (see for instance [DiSj, Chapter 7]) that $\mathcal{G}_{h}$ is bounded in $L^{2}\left(\mathbb{R}^{d} ; \mathbb{C}^{N}\right)$ and then the operators $e^{ \pm \frac{M \kappa(h)}{h}} \mathcal{G}_{h}$ are well defined.

Let $\varepsilon_{0}>0$ be small enough such that (1.7) holds on $\Sigma_{I_{\varepsilon_{0}}}:=\bigcup_{E \in I_{\varepsilon_{0}}} \Sigma_{E}$, i.e., there exists $C>0$ such that

$$
\{p, G\}(x, \xi) \geq C, \quad \forall(x, \xi) \in \Sigma_{I_{\varepsilon_{0}}},
$$

in the sense of hermitian matrices, where $I_{\varepsilon_{0}}:=\left[E_{0}-\varepsilon_{0}, E_{0}+\varepsilon_{0}\right]$. For $\eta>0$, we introduce the complex region

$$
\Gamma_{\eta}:=I_{\varepsilon_{0}}-i[0, \eta \kappa(h)]
$$

Our objective is to prove the following Lemma from which Theorem 2.5 follows.

Lemma 4.1. There exists a constant $c>0$ such that for all $M>0$, there exists $h_{M} \in(0,1]$ such that the operator $\widetilde{P}_{\theta_{1}}(h)-z$ is invertible for every $z \in \Gamma_{c M}$ and $h \in\left(0, h_{M}\right]$, and we have

$$
\left\|\left(\widetilde{P}_{\theta_{1}}(h)-z\right)^{-1}\right\|=\mathcal{O}\left(\kappa(h)^{-1}\right),
$$

uniformly for $z \in \Gamma_{c M}$ and $h \in\left(0, h_{M}\right]$.

Remark 4.2. In particular, from the above Lemma we get immediately that the operator $P_{\theta_{1}}(h)-z$ is invertible for every $z \in \Gamma_{c M}$ and $h \in\left(0, h_{M}\right]$, hence $P(h)$ has no resonances in $\Gamma_{c M}$ for all $M>0$ and $h \in\left(0, h_{M}\right]$. Furthermore, we have the following estimate on the distorted resolvent

$$
\left\|\left(P_{\theta_{1}}(h)-z\right)^{-1}\right\|=\mathcal{O}\left(h^{-C}\right),
$$

uniformly for $z \in \Gamma_{c M}$ and $h \in\left(0, h_{M}\right]$, for some constant $C>0$. 
Proof. We have

$$
\widetilde{P}_{\theta_{1}}(h)=e^{-\frac{M \kappa(h)}{h} a d_{\mathcal{G}_{h}}} P_{\theta_{1}}(h) \sim \sum_{k=0}^{+\infty} \frac{(-M \kappa(h))^{k}}{k !}\left(\frac{1}{h} \operatorname{ad}_{\mathcal{G}_{h}}\right)^{k} P_{\theta_{1}}(h),
$$

where here we use the usual notation $\operatorname{ad}_{A} B:=[A, B]$ for the commutator. The fact that $\mathcal{G}$ is scalar-valued and compactly supported ensures that $\operatorname{ad}_{\mathcal{G}_{h}} P_{\theta_{1}}(h)=\left[\mathcal{G}_{h}, P_{\theta_{1}}(h)\right]=\mathcal{O}(h)$ (in norm $\mathcal{L}\left(L^{2}\right)$ ) and then the previous asymptotic expansion makes sense since $\kappa(h) \rightarrow 0$ as $h$ tends to 0 . In particular, we have

$$
\widetilde{P}_{\theta_{1}}(h)=P_{\theta_{1}}(h)-\frac{M \kappa(h)}{h}\left[\mathcal{G}_{h}, P_{\theta_{1}}(h)\right]+\mathcal{O}\left(M^{2} \kappa(h)^{2}\right)
$$

Let $p_{\theta_{1}}, \widetilde{p}_{\theta_{1}}$ be the semiclassical symbols corresponding to $P_{\theta_{1}}(h)$ and $\widetilde{P}_{\theta_{1}}(h)$ respectively. By the $h$-pseudodifferential symbolic calculus (see for instance [DiSj, $\mathrm{Zw}]$ ), we have

$$
\widetilde{p}_{\theta_{1}}(x, \xi)=p_{\theta_{1}}(x, \xi)-i M \kappa(h)\left\{\mathcal{G}, p_{\theta_{1}}\right\}(x, \xi)+\mathcal{O}\left(M^{2} \kappa(h)^{2}\right) .
$$

On the other hand, by Taylor's expansion of $p_{\theta_{1}}$ with respect to $\theta_{1}$, we get

$$
p_{\theta_{1}}(x, \xi)=p(x, \xi)-i M \kappa(h)\{p, F(x) \cdot \xi\}(x, \xi)+\mathcal{O}\left(M^{2} \kappa(h)^{2}\right) .
$$

Combining (4.3) and (4.4), we obtain

$$
\begin{gathered}
\operatorname{Im} \widetilde{p}_{\theta_{1}}(x, \xi)=-M \kappa(h)\{p, \mathcal{G}+F(x) \cdot \xi\}(x, \xi)+\mathcal{O}\left(M^{2} \kappa(h)^{2}\right), \\
\operatorname{Re} \widetilde{p}_{\theta_{1}}(x, \xi)=p(x, \xi)+\mathcal{O}(M \kappa(h)) .
\end{gathered}
$$

According to (4.1), there exists $C>0$ such that

$$
-\operatorname{Im} \widetilde{p}_{\theta_{1}}(x, \xi) \geq C M \kappa(h), \quad \forall(x, \xi) \in \Sigma_{I_{\varepsilon_{0}}} .
$$

We write $\widetilde{P}_{\theta_{1}}(h)-z=A_{\theta_{1}}(h)-\operatorname{Re} z+i\left(B_{\theta_{1}}(h)-\operatorname{Im} z\right)$, where $A_{\theta_{1}}(h)$ and $B_{\theta_{1}}(h)$ are the self-adjoint operators given by

$$
A_{\theta_{1}}(h):=\frac{1}{2}\left(\widetilde{P}_{\theta_{1}}(h)+\left(\widetilde{P}_{\theta_{1}}(h)\right)^{*}\right), \quad B_{\theta_{1}}(h):=\frac{1}{2 i}\left(\widetilde{P}_{\theta_{1}}(h)-\left(\widetilde{P}_{\theta_{1}}(h)\right)^{*}\right) .
$$

Let $\psi_{1}, \psi_{2} \in C^{\infty}\left(\mathbb{R}^{2 d} ; \mathbb{R}\right)$ be such that, for $I \Subset I_{\varepsilon_{0}}$,

$$
\left\{\begin{array}{l}
\psi_{1}^{2}+\psi_{2}^{2}=1 \text { on } \mathbb{R}^{2 d} \\
\psi_{1}=1 \text { on } \Sigma_{I} \text { and } \operatorname{supp}\left(\psi_{1}\right) \subset \Sigma_{I_{\varepsilon_{0}}}
\end{array}\right.
$$

According to Lemma 3.2 in [SZ2], there exist two self-adjoint operators $\Psi_{1}$ and $\Psi_{2}$ with principal symbols respectively $\psi_{1}$ and $\psi_{2}$ such that

$$
\left(\Psi_{1}\right)^{2}+\left(\Psi_{2}\right)^{2}=\operatorname{Id}+\mathcal{O}\left(h^{\infty}\right) \quad \text { in } \mathcal{L}\left(L^{2}\left(\mathbb{R}^{d} ; \mathbb{C}^{N}\right)\right) .
$$

We denote by the same letters the operators $\Psi_{i}:=\Psi_{i} I_{N}, i=1,2$. On the support of $\psi_{1}$, we see from (4.6) that the principal symbol of $-B_{\theta_{1}}(h)$ is bounded from below by $C M \kappa(h)$. 
Thus, by Gårding's inequality (see e.g. [DiSj, Zw]), we have for all $u \in L^{2}\left(\mathbb{R}^{d} ; \mathbb{C}^{N}\right)$

$$
\begin{aligned}
\left\|\left(\widetilde{P}_{\theta_{1}}(h)-z\right) \Psi_{1} u\right\| \cdot\left\|\Psi_{1} u\right\| & \geq\left\langle\left(\widetilde{P}_{\theta_{1}}(h)-z\right) \Psi_{1} u, \Psi_{1} u\right\rangle \\
& \geq\left\langle\left(\operatorname{Im} \widetilde{P}_{\theta_{1}}(h)-\operatorname{Im} z\right) \Psi_{1} u, \Psi_{1} u\right\rangle \\
& =\left\langle\left(\operatorname{Im} z-B_{\theta_{1}}(h)\right) \Psi_{1} u, \Psi_{1} u\right\rangle \\
& \geq(\operatorname{Im} z+C M \kappa(h)-\mathcal{O}(h))\left\|\Psi_{1} u\right\|^{2} \\
& \geq \frac{C}{3} M \kappa(h)\left\|\Psi_{1} u\right\|^{2},
\end{aligned}
$$

uniformly for $\Im z>-\frac{C}{3} M \kappa(h)$.

On the other hand, since $A_{\theta_{1}}(h)-\operatorname{Re} z$ is uniformly elliptic on the support of $\psi_{2}$ and $\operatorname{Re} z \in I_{\varepsilon_{0}}$, the symbolic calculus permits us to construct a parametrix $R \in \mathcal{S}^{0}\left(\langle\xi\rangle^{-2}\right)$ of $A_{\theta_{1}}(h)-\operatorname{Re} z$ such that, in the sense of corresponding symbols,

$$
R \#\left(A_{\theta_{1}}(h)-\operatorname{Re} z\right) \psi_{2}=\psi_{2}+\mathcal{O}\left(h^{\infty}\right),
$$

where \# stands for the Weyl composition of symbols. As a consequence, we obtain

$$
\left\|\left(\widetilde{P}_{\theta_{1}}(h)-z\right) \Psi_{2} u\right\| \geq \frac{1}{C^{\prime}}\left\|\Psi_{2} u\right\|-\mathcal{O}\left(h^{\infty}\right)\|u\|^{2} .
$$

for all $u \in L^{2}\left(\mathbb{R}^{d} ; \mathbb{C}^{N}\right)$. Furthermore, by means of standard elliptic arguments, one can easily prove the following semiclassical inequality, for $i=1,2$,

$$
\left\|\left[\widetilde{P}_{\theta_{1}}(h), \Psi_{i}\right] u\right\| \leq C_{2} h\left(\left\|\widetilde{P}_{\theta_{1}}(h) u\right\|+\|u\|\right), \quad \forall u \in H^{2}\left(\mathbb{R}^{d} ; \mathbb{C}^{N}\right) .
$$

Combining (4.8), (4.9), (4.10), and (4.11) with the estimate

$$
\begin{gathered}
\left\|\left(\widetilde{P}_{\theta_{1}}(h)-z\right) u\right\|^{2}=\sum_{i=1}^{2}\left\|\Psi_{i}\left(\widetilde{P}_{\theta_{1}}(h)-z\right) u\right\|^{2}-\mathcal{O}\left(h^{\infty}\right)\left\|\left(\widetilde{P}_{\theta_{1}}(h)-z\right) u\right\|^{2} \\
\geq \frac{1}{2} \sum_{i=1}^{2}\left\|\left(\widetilde{P}_{\theta_{1}}(h)-z\right) \Psi_{i} u\right\|^{2}-\sum_{i=1}^{2}\left\|\left[\widetilde{P}_{\theta_{1}}(h), \Psi_{i}\right] u\right\|^{2}-\mathcal{O}\left(h^{\infty}\right)\left\|\left(\widetilde{P}_{\theta_{1}}(h)-z\right) u\right\|^{2},
\end{gathered}
$$

we deduce, for $z \in \Gamma_{c M}:=\left\{z \in \mathbb{C} ; \operatorname{Re} z \in I_{\varepsilon_{0}}\right.$ and $\left.\operatorname{Im} z \geq-c M \kappa(h)\right\}$ (with $c>0$ independent of $M$ and $h$ ) and sufficiently small $h$,

$$
\left\|\left(\widetilde{P}_{\theta_{1}}(h)-z\right) u\right\| \geq \frac{\kappa(h)}{C}\|u\| .
$$

By the same arguments, we prove an estimate similar to (4.13) for the adjoint operator $\left(\widetilde{P}_{\theta_{1}}(h)\right)^{*}-\bar{z}$ and we conclude that $\widetilde{P}_{\theta_{1}}(h)-z$ is invertible for every $z \in \Gamma_{c M}$. Hence, $\widetilde{P}_{\theta_{1}}(h)$ has no spectrum in $\Gamma_{c M}$ and we have the estimate

$$
\left\|\left(\widetilde{P}_{\theta_{1}}(h)-z\right)^{-1}\right\| \leq C \kappa(h)^{-1}
$$

uniformly for $z \in \Gamma_{c M}$. 
4.2. Proof of Theorem 2.3. We start by proving the following estimate on the distorted resolvent.

Lemma 4.3. Assume $\left(\mathbf{H o l}_{\infty}\right)$ and let $\left\|V_{\infty}\right\|_{N \times N}<\alpha<\beta<+\infty, \eta>0$ and $\theta=h|\ln h|$. Then, there exist a constant $C>0$ and $h_{0} \in(0,1]$ such that

$$
\left\|\left(P_{\theta}(h)-z\right)^{-1}\right\|=\mathcal{O}\left(e^{C / h}\right),
$$

uniformly for $z \in[\alpha, \beta]-i[0, \eta h|\ln h|], z \notin \operatorname{Res}(P(h))$ and $h \in\left(0, h_{0}\right]$.

Proof. Let $\psi \in C_{0}^{\infty}\left(\mathbb{R}^{d} ;[0,1]\right)$ be such that $\psi(x)=1$ for $|x| \leq 1$ and $\psi(x)=0$ for $|x| \geq 2$. We introduce the Schrödinger operator

$$
\mathcal{A}(h):=-h^{2} \Delta \cdot I_{N}+V_{r}(x), \quad \text { with } \quad V_{r}(x):=\left(1-\psi\left(\frac{x}{r}\right)\right) V(x), \quad r>0 .
$$

Let $a_{r}(x, \xi):=|\xi|^{2} I_{N}+V_{r}(x)$ be the semiclassical symbol of $\mathcal{A}(h)$ and set

$$
\Sigma_{E}^{r}:=\bigcup_{j=1}^{N}\left\{(x, \xi) \in \mathbb{R}^{2 d} ;|\xi|^{2}+\left(1-\psi\left(\frac{x}{r}\right)\right) \lambda_{j}(x)=E\right\}, \quad \Sigma_{[\alpha, \beta]}^{r}:=\bigcup_{E \in[\alpha, \beta]} \Sigma_{E}^{r} .
$$

where we recall that the $\lambda_{j}(x)$ 's are the eigenvalues of $V(x), x \in \mathbb{R}^{d}$. From $\left(\mathbf{H o l}_{\infty}\right)$, for $r>0$ large enough, we have $|\xi|^{2}>\alpha / 2$ on $\pi_{\xi}\left(\Sigma_{[\alpha, \beta]}^{r}\right)$. Here $\pi_{\xi}$ denotes the spatial projection $(x, \xi) \mapsto \xi$. On the other hand, using that $x \cdot \nabla_{x} V(x) \rightarrow+\infty$ as $|x| \rightarrow+\infty$ according to $\left(\mathbf{H o l}_{\infty}\right)$ and the Cauchy formula, we get

$$
\begin{aligned}
\left\{a_{r}, x \cdot \xi\right\}(x, \xi) & =2|\xi|^{2} I_{N}+\frac{x}{r} \nabla_{x} \psi\left(\frac{x}{r}\right) V(x)-\left(1-\psi\left(\frac{x}{r}\right)\right) x \nabla_{x} V(x) \\
& \geq \alpha / 2,
\end{aligned}
$$

for $(x, \xi) \in \Sigma_{[\alpha, \beta]}^{r}$ and $r>0$ large enough. Thus, we deduce that $\mathbb{R}^{2 d} \ni(x, \xi) \mapsto x \cdot \xi$ is an escape function associated with $a_{r}$ on $\Sigma_{[\alpha, \beta]}^{r}$ for $r>0$ large enough.

Let $\mathcal{A}_{\theta}(h)$ be the distorted operator associated with $\mathcal{A}(h)$, obtained by replacing $P(h)$ with $\mathcal{A}(h)$ in (2.10). Let $\chi \in C_{0}^{\infty}\left(\mathbb{R}^{d} ;[0,1]\right)$ such that $\chi(x)=1$ for $|x|<2 r$. In particular, we have

$$
\chi\left(V-V_{r}\right)=\chi \psi_{r} V=\left(V-V_{r}\right) .
$$

Using the resolvent identity, we get for $z \notin \operatorname{Res}(P(h))$,

$$
\begin{aligned}
\left(P_{\theta}(h)-z\right)^{-1} & =\left(\mathcal{A}_{\theta}(h)-z\right)^{-1}-\left(P_{\theta}(h)-z\right)^{-1}\left(V-V_{r}\right)\left(\mathcal{A}_{\theta}(h)-z\right)^{-1} \\
& =\left(\mathcal{A}_{\theta}(h)-z\right)^{-1}-\left(\mathcal{A}_{\theta}(h)-z\right)^{-1}\left(V-V_{r}\right)\left(\mathcal{A}_{\theta}(h)-z\right)^{-1} \\
& +\left(\mathcal{A}_{\theta}(h)-z\right)^{-1}\left(V-V_{r}\right)\left(P_{\theta}(h)-z\right)^{-1}\left(V-V_{r}\right)\left(\mathcal{A}_{\theta}(h)-z\right)^{-1} \\
& =\left(\mathcal{A}_{\theta}(h)-z\right)^{-1}-\left(\mathcal{A}_{\theta}(h)-z\right)^{-1}\left(V-V_{r}\right)\left(\mathcal{A}_{\theta}(h)-z\right)^{-1} \\
& +\left(\mathcal{A}_{\theta}(h)-z\right)^{-1}\left(V-V_{r}\right) \chi(P(h)-z)^{-1} \chi\left(V-V_{r}\right)\left(\mathcal{A}_{\theta}(h)-z\right)^{-1} .
\end{aligned}
$$

According to the non-trapping estimate (4.2), there exists a constant $C^{\prime}>0$ such that

$$
\left\|\left(\mathcal{A}_{\theta}(h)-z\right)^{-1}\right\|=\mathcal{O}\left(h^{-C^{\prime}}\right),
$$

uniformly for $z \in[\alpha, \beta]-i[0, \eta h|\ln h|]$ and $h>0$ small enough. It follows that

$$
\left\|\left(P_{\theta}(h)-z\right)^{-1}\right\|=\mathcal{O}\left(h^{-2 C^{\prime}}+h^{-2 C^{\prime}}\left\|\chi(P(h)-z)^{-1} \chi\right\|\right),
$$


uniformly for $z \in[\alpha, \beta]-i[0, \eta h|\ln h|], z \notin \operatorname{Res}(P(h))$ and $h>0$ small enough. On the other hand, the weighted estimate (2.4) clearly implies the same estimate for the truncated resolvent, that is

$$
\left\|\chi(P(h)-z)^{-1} \chi\right\|=\mathcal{O}\left(e^{C^{\prime \prime} / h}\right),
$$

for some constant $C^{\prime \prime}>0$, uniformly for $z \in[\alpha, \beta]-i[0, \eta h|\ln h|]$ and $h>0$ small enough. Putting together (4.17) and (4.18), we obtain the desired estimate (4.15).

End of the proof of Theorem 2.3. Let $J=[\alpha, \beta] \subset\left(\left\|V_{\infty}\right\|_{N \times N},+\infty\right)$ and let $\theta=h|\ln h|$. For $z \in \mathbb{C}$ with $\operatorname{Re} z \in J$, we write

$$
P_{\theta}(h)-z=\left(P_{\theta}(h)-\operatorname{Re} z\right)(I-K(z ; h)) \quad \text { with } \quad K(z ; h):=i \operatorname{Im} z\left(P_{\theta}(h)-\operatorname{Re} z\right)^{-1} .
$$

According to Lemma 4.3, there exists a constant $C>0$ such that for $h$ small enough,

$$
\left\|\left(P_{\theta}(h)-\operatorname{Re} z\right)^{-1}\right\| \leq C e^{C / h} .
$$

It follows that for $h$ small enough, $\operatorname{Re} z \in J$ and $|\operatorname{Im} z|<\frac{1}{C} e^{-C / h}$, we have

$$
\|K(z ; h)\| \leq C e^{C / h}|\operatorname{Im} z|<1 \text {. }
$$

Therefore $P_{\theta}(h)-z$ is invertible for $z \in J-i\left[0,-\frac{1}{C} e^{-C / h}\right]$, hence $P(h)$ has no resonances in this region. This ends the proof of Theorem 2.3.

\section{ACKNOWLEDGMENTS}

The author thanks Gilles Lebeau for valuable discussions about Carleman estimates at Université de Nice in 2018. The author also thanks Jean-François Bony and Claudio Fernández for many stimulating remarks. The research of the author was supported by CONICYT FONDECYT Grant No. 3180390.

\section{REFERENCES}

[Ag] S. Agmon, Spectral properties of Schrödinger operators and scattering theory, Ann., Scuola Norm., sup., Pisa, (4)2 (1975), 151-218

[AC] J. Aguilar, J. M. Combes, A class of analytic perturbations for one-body Schrödinger Hamiltonians, Comm. Math. Phys., 22 (1971) 269-279.

[Ash] S. Ashida: Molecular predissociation resonances below an energy level crossing. Asymptot. Anal. 107 (2018), no. 3-4, 135-167.

[As] M. Assal, Semiclassical resolvent estimates for Schrödinger operators with matrix-valued potentials and applications, work in progress.

[ADF] M. Assal, M. Dimassi, S. Fujiı́, Semiclassical trace formula and spectral shift function for systems via a stationary approach, Int. Math. Res. Notices, Vol. 2019, No. 4, pp. 1227-1264.

[Bu] N. BuRQ, Lower bounds for shape resonances widths of long range Schrödinger operators, American Journal of Mathematics, Volume 124, Number 4, 2002, pp. 677-735.

[CV] F. CARdoso, G. Vodev, Uniform estimates of the resolvent of the Laplace-Beltrami operator on infinite volume Remannian manifolds II, Annals of Henri Poincaré, (4)3 (2002), 673-691.

[DiSj] M. Dimassi, J. SJöstrand, Spectral asymptotics in the semi-classical limit, London Mathematical Society, Lecture Note Series 268, 1999.

[Da] K. Datchev, Quantitative limiting absorption principle in the semiclassical limit, Geom. Funct. Anal. Vol. 24 (2014) 740-747.

[DDZ] K. Datchev, S. Dyatlov, M. Zworski, Resonances and lower resolvent bounds, J. of. Spectral Theory, 5(3) (2015), 599-615. 
[FMW1] S. Fujié, A. Martinez, T. WATAnABe, Widths of resonances at an energy-level crossing I: Elliptic interaction, J. Diff. Eq. 260 (2016) 4051-4085.

[FMW2] S. Fujié, A. Martinez, T. WATAnABE, Widths of resonances at an energy-level crossing II: Vector field interaction, J. Diff. Eq. 262 (2017) 5880-5895.

[FMW3] S. Fujiı́, A. MARTinez, T. WATAnABE, Widths of resonances above an energy-level crossing, Preprint, arxiv: 1904.12511.

[GM] C. GÉRARd, A. MARTinez, Principe d'absorption limite pour les opérateurs de Schrödinger à longue portée, C. R. Acad. sci. Paris 306 (1988), 121-123.

[HM] B. Helffer, A. Martinez, Comparaison entre les diverses notions de résonances, Helv. Phys. Acta 60 (1987), no. 8, 992-1003.

[Hi] K. Higuchi, Resonances free domain for a system of Schrödinger operators with energy-level crossings, Preprint, arxiv: 1912.10180.

[Hu] W. Hunziker, Distortion analyticity and molecular resonance curves, Ann. Inst. Henri Poincare, Phys. Theor. 45, 339-358 (1986).

[Je1] T. JEcko, On the mathematical treatment of the Born-Oppenheimer approximation, Journal of Mathematical Physics 55, (2014).

[Je2] T. JECKo, Estimations de la résolvante pour une molécule diatomique dans l'approximation de BornOppenheimer, Comm. Math. Phys., 195(3), 585-612., 1998.

[Je3] T. Jеско, Semiclassical resolvent estimates for Schrödinger matrix operators with eigenvalues crossings, Math. Nachr., 257, 1, p. 36-54, 2003.

[Je4] T. JECKO, Non-trapping condition for semiclassical Schrödinger operators with matrix-valued potentials, Math. Phys. Electronic Journal, 11(2), 2005.

[FR] C. Fermanian-Kammerer, V. Rousse, Resolvent Estimates and Matrix-Valued Schrödinger Operator with Eigenvalue Crossings; Application to Strichartz Estimates, Comm. in PDE, 33 (2008), 19-44.

[Ka] T. Kato, Perturbation Theory for Linear Operators, Berlin: Springer, 1995.

[KMSW] M. Klein, A. Martinez, R. Seiler, X. P. WAng, On the Born-Oppenheimer expansion for polyatomic molecules, Comm. Math. Phys. 143 (1992), 607-639.

[Ma] A. Martinez, Resonance Free Domains for Non Globally Analytic Potentials, Ann. Henri Poincaré 4 2002, 736-759.

[Mi] L. Michel, Semi-Classical Behavior of the Scattering Amplitude for Trapping Perturbations at Fixed Energy, Canad. J. Math., Vol. 56(4), 2004, 794-829.

[Na] S. NAKAmurA, Scattering theory for the shape of resonance model I, II, Ann. IHP Vol. 50, 1989, 35-52 and 53-62.

[NSZ] S. Nakamura, P. Stefanov, M. Zworski, Resonance expansions of propagators in the presence of potential barriers, J. Func. Anal., 205, (2003) 180-205.

[Ne] L. Nedelec, Resonances for matrix Schrödinger operators, Duke Math. J., Vol. 106(2), 2001.

[PZ] V. Petkov, M. Zworski, Semi-Classical estimates on the scattering determinant, Ann. Henri Poincaré, $2(2001), 675-711$.

[RT] D. Robert, H. TAmura, Semiclassical estimates for resolvant and asymptotics for the total scattering cross-sections, Ann. IHP Phys. Théor., 46(4) (1987), 415-442.

[RoTa] I. Rodianski, T. Tao, Effective Limiting Absorption Principles, and Applications, Commun. Math. Phys. 333, 1-95 (2015).

[Sh] J. Shapiro, Semiclassical resolvent bounds in dimension two, to appear in Proceedings of the American Mathematical Society, arXiv 1604.038452.

[Sj] J. SJÖstRAND, Lectures on resonances, http://sjostrand.perso.math.cnrs.fr/Coursgbg.pdf, 2002.

[SZ1] J. Sjöstrand, M. Zworski, Complex scaling and the distribution of scattering poles, J. Amer. Math. Soc., 4 (1991), 729-769.

[SZ2] J. Sjöstrand, M. Zworski, Fractal upper bounds on the density of semiclassical resonances, Duke Math. J., 137, no. 3 (2007), 381-459.

[Vo1] G. Vodev, On the exponential bound of the cutoff resolvent, Serdica Math. J., 26, (2000), 49-58.

[Zw] M. Zworski, Semiclassical Analysis, Graduate studies in mathematics 138, 2012. 
Marouane Assal, Facultad de Matemáticas, Pontificia Universidad Católica de Chile, Vicuna Mackenna 4860, Santiago De Chile

E-mail address: marouane.assal@mat.uc.cl 